Original paper

\title{
A Monte Carlo investigation of the spatial resolution performance of a small-animal PET scanner designed for mouse brain imaging studies
}

\author{
Mercedes Rodríguez-Villafuerte ${ }^{\mathrm{a}, \mathrm{b}, *, 1}$, Yongfeng Yang ${ }^{\mathrm{b}}$, Simon R. Cherry ${ }^{\mathrm{b}}$ \\ a Instituto de Física, Universidad Nacional Autónoma de México, A. P. 20-364, 01000 Mexico D. F., Mexico \\ ${ }^{\mathrm{b}}$ Department of Biomedical Engineering, University of California-Davis, One Shields Avenue, Davis, CA 95616, USA
}

\section{A R T I C L E I N F O}

\section{Article history:}

Received 5 December 2012

Received in revised form

27 February 2013

Accepted 13 March 2013

Available online 6 April 2013

\section{Keywords:}

Discrete cosine transform

High-resolution imaging

Monte Carlo

Small-animal PET

\begin{abstract}
A B S T R A C T
Our laboratory has developed PET detectors with depth-encoding accuracy of $\sim 2 \mathrm{~mm}$ based on finely pixelated crystals with a tapered geometry, readout at both ends with position-sensitive avalanche photodiodes (PSAPDs). These detectors are currently being used in our laboratory to build a one-ring high resolution PET scanner for mouse brain imaging studies. Due to the inactive areas around the PSAPDs, large gaps exist between the detector modules which can degrade the image spatial resolution obtained using analytical reconstruction with filtered backprojection (FBP). In this work, the Geant4based GATE Monte Carlo package was used to assist in determining whether gantry rotation was necessary and to assess the expected spatial resolution of the system. The following factors were investigated: rotating vs. static gantry modes with and without compensation of missing data using the discrete cosine transform (DCT) method, two levels of depth-encoding, and positron annihilation effects for ${ }^{18} \mathrm{~F}$. Our results indicate that while the static scanner produces poor quality FBP images with streak and ring artifacts, the image quality was greatly improved after compensation of missing data. The simulation indicates that the expected FWHM system spatial resolution is $0.70 \pm 0.05 \mathrm{~mm}$, which approaches the predicted limit of $0.5 \mathrm{~mm}$ FWHM due to positron range, photon non-colinearity and physical detector element size effects. We conclude that excellent reconstructed resolution without gantry rotation is possible even using FBP if the gaps are appropriately handled and that this design can approach the resolution limits set by positron annihilation physics.
\end{abstract}

(c) 2013 Associazione Italiana di Fisica Medica. Published by Elsevier Ltd. All rights reserved.

\section{Introduction}

Dedicated positron emission tomography (PET) scanners for small-animal studies have been under investigation for the last 20 years. It is estimated that over 250 dedicated small-animal PET scanners are installed worldwide, with typical spatial resolutions in the 1-2 $\mathrm{mm}$ range. Unfortunately, the performance of these scanners is not sufficient to carry out quantitative studies of structures within the mouse brain where the targeted volume of the entire organ is only $0.5 \mathrm{~cm}^{3}$. According to Larobina et al. [1] a scanner with spatial resolution of $\sim 0.4 \mathrm{~mm}$ is required to achieve mouse brain images comparable with those produced by a clinical PET scanner with $5 \mathrm{~mm}$ spatial resolution for the human brain. Simulation studies have shown that the best resolution that can be achieved

\footnotetext{
* Corresponding author. Instituto de Física, Universidad Nacional Autónoma de México, Experimental Physics, A. P. 20-364, 01000 Mexico D. F., Mexico. Tel.: +52 55 5622 5052; fax: +525556161535.

E-mail address: mercedes@fisica.unam.mx (M. Rodríguez-Villafuerte).

1 On sabbatical leave from Instituto de Física.
}

for small-animal PET is of the order of $0.5 \mathrm{~mm}$ when using very small scintillator elements [2].

One of the major challenges to develop a PET scanner with such high spatial resolution is to maintain image quality in terms of signal-to-noise ratio. This implies that the sensitivity of the scanner must increase as the spatial resolution improves to avoid large increases in scan time and injected radioactivity. There is a tradeoff between sensitivity and resolution that simultaneously requires the use of thicker crystals and innovative geometries to improve sensitivity, and detectors that provide access to the depth-of-interaction (DOI) information to avoid degradation in spatial resolution due to parallax errors. The use of DOI detectors is particularly important for small diameter scanners.

Several groups have proposed detector designs based on different DOI encoding approaches to achieve sub-millimeter spatial resolution for small-animal PET systems [3-9]. Our group has pursued detectors with good depth-encoding accuracy of $\sim 2 \mathrm{~mm}[10,11]$ and small scintillation crystal cross-sections [2,12-14] suitable to build an animal PET scanner with a small ring diameter that can simultaneously obtain high spatial resolution and high sensitivity. 
The design of our detectors involves dual-ended readout of finely pixelated scintillator arrays with position-sensitive avalanche photodiodes (PSAPDs). Improvement in the solid angle coverage is achieved by using a novel tapered geometry of the crystal elements, with a trapezoidal shape in the transaxial direction and rectangular shape in the axial direction. Using this detector configuration, a prototype one-ring PET scanner for mouse brain studies is currently being built in our laboratory. The scanner consists of sixteen detector modules arranged in a $61 \mathrm{~mm}$ inner diameter ring, each detector composed of crystal elements with cross-section of $0.45 \mathrm{~mm}$ at the front face. Since there are unavoidable inactive areas at the edge of the PSAPDs, there are significant gaps between detector modules that produce zero and low count elements in the sinograms. The missing data can result in streaking artifacts in the reconstructed images when using methods that require a complete data set, such as the analytical 2D filtered backprojection (FBP) algorithm, and in iterative algorithms will likely lead to a loss in spatial resolution. There are two important reasons for wanting a scanner design that can produce high-quality reconstructions using FBP-based methods. The first relates to the fact that analytic algorithms are linear and, thereby, allow easier control of the spatial resolution and noise properties in the reconstruction, which is important for quantitative data analysis [15]. The second reason, and probably the most relevant, is that FBP is required for carrying out scanner performance protocols such as the international NEMA NU-4 2008 standards [16], proposed as a standardized methodology for the evaluation of dedicated PET scanners for animal imaging.

Photon non-colinearity and positron range are normally classified as the unavoidable physical limitations of spatial resolution in PET [17], and given the desired spatial resolution of our scanner, these effects play an important role. The non-colinearity blurring is on the order of $0.14 \mathrm{~mm}$ for a $6.1 \mathrm{~cm}$ diameter scanner. It increases linearly with scanner diameter, thus bore size should be minimized to limit the effect. The isotope-dependent positron range effect has a contribution that also requires careful examination [2]. For the low energy positron emitter fluorine- $18\left({ }^{18} \mathrm{~F}\right)$, the most widely used radionuclide in PET, the positron spectrum has an end-point energy of $635 \mathrm{keV}$, with $250 \mathrm{keV}$ mean energy. While the average range of the $\beta^{+}\left[{ }^{18} \mathrm{~F}\right]$ positrons in water is $0.64 \mathrm{~mm}$, their root mean square effective range is $\sim 0.2 \mathrm{~mm}$ [18]. Since both these factors are within the same order of magnitude as the size of our crystal elements, positron annihilation physics will limit the spatial resolution of our proposed small-animal PET scanner and quantification of the effects is necessary.

The Monte Carlo (MC) method has become an essential tool in PET to assist detector design, to optimize acquisition and processing protocols as well as to predict the performance of systems being developed. The MC techniques are useful in those studies where experimental measurements are difficult to implement. For instance, while the positron annihilation effects on the spatial resolution of a PET scanner are complex to measure due to the finite detector resolution, positron transport in matter can be modeled accurately using MC and applied to quantify such effects. Another good example where MC can be extremely valuable is in the evaluation of the image quality for a scanner whose geometry results in gaps, like the ones produced with our system. There are two possible approaches to fill the gaps, the first one would require rotating the scanner gantry during data acquisition. However, designing and implementing a gantry to rotate the detectors can be cumbersome, time consuming, expensive and, quite possibly, unnecessary. It is here where the simulation of gantry rotation can provide useful information and be used as a guideline to plan the image acquisition protocol and gantry design. The second approach would involve the implementation of a numerical gap-filling algorithm to compensate for missing data produced with a static scanner. In this case, the performance of gap-filling methods can be easily investigated with static-mode data produced with the simulation.

In this work Monte Carlo simulation studies were used to assess the reconstructed spatial resolution performance of a proposed one-ring small-animal PET scanner for mouse brain studies. The following factors were investigated (Fig. 1) for point sources across the scanner field of view (FOV) and an image resolution hot-rod phantom:

\section{Gantry acquisition:}

1.1. Static-mode that produced sinograms with missing data due to the presence of gaps between the detectors.

1.2. Static-mode with gap-filling compensation (Static GF) using an iterative procedure based on the application of a data adaptive filter in the discrete cosine transform domain.

1.3. Rotating-mode assuming a step-and-shoot scheme, with four angular steps to completely calculate all lines of response.

2. Positron annihilation physics. Two source types were simulated assuming a pair of anti-parallel $511 \mathrm{keV}$ photons and a realistic positron source that follows the $\left[{ }^{18} \mathrm{~F}\right]$ emission spectrum.

3. Depth-encoding accuracy. The analysis included the DOI resolution of the PET detectors using a Gaussian distribution with FWHM $_{\text {DOI }}$ of either $2 \mathrm{~mm}$ or $3 \mathrm{~mm}$ as the blurring kernel.

The simulation of the gantry rotation was used to examine the effect of the gaps on reconstructed images and to determine whether it is necessary to build a rotating gantry for the scanner. The combined simulation of positron annihilation physics together with a realistic geometry of the scanner that incorporates the DOI resolution accuracy of our detectors was used to investigate the best spatial resolution that we could expect to achieve with our small-animal scanner using the standard FBP analytic reconstruction algorithm. We choose to focus on reconstruction with the FBP algorithm, as this is required for NEMA spatial resolution protocols [16] that ultimately will be used to measure scanner performance, and also because the effect of the detector gaps will likely be most significant for analytic based reconstruction techniques. Since the scanner consists of a single ring of detector modules, the studies will examine the reconstructed spatial resolution in the central transaxial plane.

\section{Materials and methods}

\section{Scanner configuration}

The geometry of the simulated small-animal PET scanner is shown in Fig. 2. The system is composed of 16 detectors arranged in

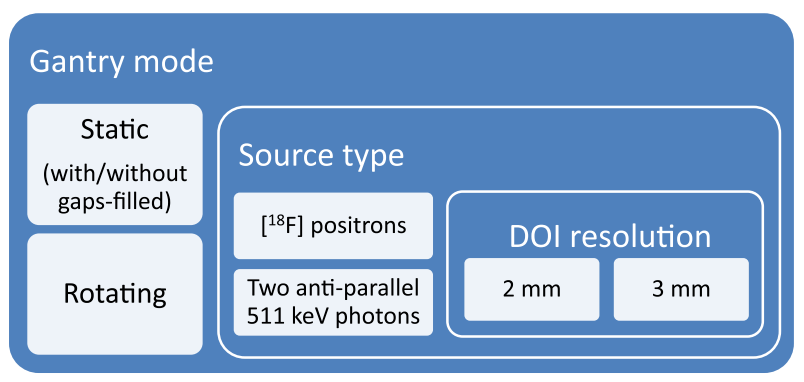

Figure 1. Nested diagram showing the combined parameters used in the MC to investigate the reconstructed spatial resolution of the small-animal scanner for mouse brain studies. 


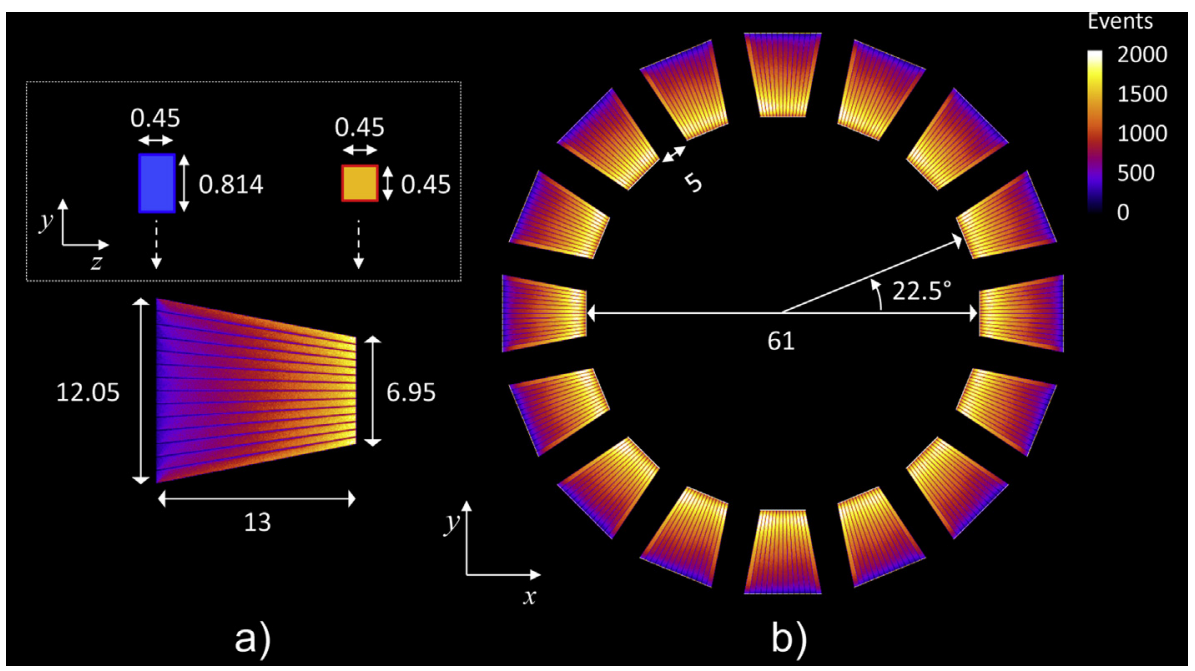

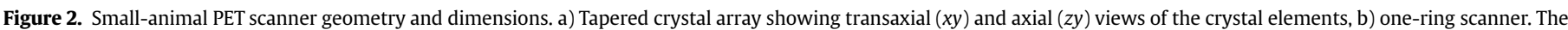
color scale indicates the relative number of $511 \mathrm{keV}$ photons interacting in the detectors for a central extended source. All distances are in mm.

a single ring of $6.95 \mathrm{~mm}$ axial length, and inner and outer diameter of 61 and $87 \mathrm{~mm}$, respectively. The detector modules are based on DOI-encoding detectors with dual-ended readout at the front and back ends of scintillator crystal arrays by PSAPDs [10,11]. For each registered event, a continuous measure of DOI is achieved by taking the ratio of the signals from both photodetectors. This configuration, designed and optimized by our group over several years, shows only a slight dependence of DOI resolution with depth, with average FWHM $_{\text {DoI }}$ values between 2 and $3 \mathrm{~mm}$ [11].

Each detector has a $14 \times 14$ array of lutetium oxyorthosilicate (LSO) crystals separated by a $50 \mu \mathrm{m}$ thick reflector; the crystal array has been designed with a tapered geometry to increase sensitivity [14]. At the entrance face of the detector the crystal elements have dimensions of $0.45 \times 0.45 \mathrm{~mm}^{2}$ with a pitch of $0.5 \mathrm{~mm}$, while at the far end the crystals are $0.45 \times 0.814 \mathrm{~mm}^{2}$ with a pitch of $0.5 \mathrm{~mm}$ in the axial direction and $0.864 \mathrm{~mm}$ transaxially. The length of the crystal elements varies within the trapezoidal shape of the array from $13 \mathrm{~mm}$ at the center to $13.25 \mathrm{~mm}$ at the edge. The efficiencies for these crystal lengths for $511 \mathrm{keV}$ photons (no energy threshold) are greater than $60 \%$.

The angular separation between the detector modules was chosen to minimize the gaps between the PSAPDs. Our prototype scanner uses PSAPDs of different areas to conform to the crystal array shape; $10 \times 10 \mathrm{~mm}^{2}$ at the front and $10 \times 15.2 \mathrm{~mm}^{2}$ at the back. The physical size of these photodetectors leads to a $22.5^{\circ}$ angular interval between the modules, which results in the presence of significant air gaps between the crystal arrays.

Both static and step-and-shoot rotating modes were simulated to examine the effect of the gaps on the spatial resolution. The latter (which will be considered as the reference) considered four angular steps at $0^{\circ}, 5.625^{\circ}, 11.25^{\circ}$ and $16.875^{\circ}$, sufficient to completely fill the gaps in the sinogram space.

\section{Monte Carlo simulation and data post-processing}

The Geant4 Application for Emission Tomography (GATE) simulation code [19] was used in this work. GATE allows the coupled simulation transport of ionizing radiation (electrons, photons, positrons and ions) over a wide energy range and in arbitrary materials. Additionally, GATE offers the possibility of describing complex scanner geometries (that can include source or detector movement), modeling radioactive emissions and physics processes, and simulating the signal-processing-chain response.
The geometry of the proposed small-animal scanner was incorporated in GATE. Coincidence events were registered assuming a timing coincidence window of $20 \mathrm{~ns}$, an energy resolution of $25 \%$, an energy window of $150-750 \mathrm{keV}$, and each detector in coincidence with eleven opposing detectors. The energy and timing resolutions were selected based on reported values for similar detectors $[10,13]$. Using a wide energy window is indicated in the case of small-animal studies where the object scatter is low [20]. Low-activity sources were assumed in all the simulated studies to minimize the contribution of random events. Because this study is focused on estimations of spatial resolution, effects of deadtime and events due to ${ }^{176} \mathrm{Lu}$ background were not simulated.

The experimental DOI encoding accuracy was simulated by following the procedure reported by St. James et al. [14]. From the simulations, the energy-weighted $(x, y, z)$ position of interaction in the scintillator was extracted as well as the crystal element identification for every recorded coincidence event. The finite DOI detector resolution was modeled by applying a random Gaussian blurring to the $(x, y)$ position along the depth direction of the crystal element where the interaction took place. For tapered crystal arrays, every crystal element has a different orientation defined by its long axis. This was taken into account to perform the DOI blurring along the individual crystal axis direction. The standard deviation of the Gaussian distribution was chosen as FWHM $_{\text {DoI }} / 2.35$. In those cases where the random blurring procedure placed the interaction outside the crystal boundaries, the new position was selected to be either the front or back face of the crystal as appropriate. In order to avoid artificial sampling nonuniformities in the sinograms (caused by all events being discretized in $(x, y)$ by physical crystal dimensions), an additional uniform random blurring was applied wherein the events were randomly positioned across the transverse width of the crystal rather than at the center of the crystal. All calculations considered two possible values for the $\mathrm{FWHM}_{\mathrm{DOI}}(2$ and $3 \mathrm{~mm}$ ) which encompasses the expected DOI resolution capabilities of our prototype scanner [11].

The blurred-positions were then transformed into raw sinograms of 600 angles and 300 radial positions, with bin dimensions of $\Delta \phi=0.3^{\circ}$ and $\Delta s=0.15 \mathrm{~mm}$, respectively. These sinograms were normalized as described in the following section. The tomographic images were reconstructed by conventional 2D FBP with a SheppLogan filter with cutoff at the Nyquist frequency. A $282 \times 282$ image matrix was used with $0.15 \mathrm{~mm}$ pixel size. 


\section{Normalization sinogram}

In order to correct the raw sinograms for variations in crystal efficiencies and geometric effects, a simple normalization procedure was applied to all simulated studies, based on direct inversion of a sinogram simulated with a uniform cylindrical phantom covering the transverse and axial FOV of the small-animal scanner. Low activity (400 kBq) isotropic point sources of two anti-parallel $511 \mathrm{keV}$ photons generated at $180^{\circ}$ opposing directions and uniformly distributed in a non-attenuating cylinder of $25 \mathrm{~mm}$ radius and $8 \mathrm{~mm}$ height was considered. For all the registered lines of response (LOR) a path length correction was applied to correct for non-uniform radial illumination of the detector pairs. The normalization coefficients were calculated as the number of counts in each LOR divided by the average number of coincidence events. Taking advantage of the rotational symmetry in the scanner geometry, data statistics were improved by 8 -fold by adding the matrix elements in $22.5^{\circ}$ intervals. As a result of this procedure, more than 130 million coincidence events were obtained for the normalization sinogram. Different normalization sinograms were formed for each DOI resolution (2 and $3 \mathrm{~mm}$ ) and scanner mode acquisition (static or rotating). The sinograms were normalized by dividing them on an element by element basis with the appropriate normalization sinogram.

\section{Reconstructed resolution}

The reconstructed spatial resolution of the small-animal PET scanner across its FOV was estimated by simulating ideal isotropic point sources ( $400 \mathrm{kBq}$ ) at different radial offsets (from 0 to $14 \mathrm{~mm}$ in $2 \mathrm{~mm}$ steps) assuming $200 \mathrm{~s}$ scanner acquisition duration per radial position. Two types of sources were simulated, two antiparallel $511 \mathrm{keV}$ photons and a positron source that follows the $\beta^{+}\left[{ }^{18} \mathrm{~F}\right]$ spectrum. In the latter case, in order to ensure the complete annihilation of positrons, the point source was assumed to be embedded in an acrylic cube of $10 \mathrm{~mm}$ extent on all sides, as recommended by the NEMA NU-4 protocol [16]. The raw sinograms were formed with 5 million coincidence events (on average) per radial position. After normalization, images were reconstructed by conventional 2D FBP following the procedure described previously, and profiles through the peak in each reconstructed image were extracted. The spatial resolution along the radial and tangential directions was analyzed in terms of the FWHM following the NEMA NU 4-2008 standards [16]. In this method, the FWHM was determined by linear interpolation between adjacent pixels at half the maximum value of the profile. The maximum value was obtained by a parabolic fit using the peak point and its two nearest neighboring points.

\section{Image resolution phantom}

To assess the image resolution of the small-animal PET scanner, a simulation of a microDerenzo phantom was carried out with hollow channel sizes relevant to the expected spatial resolution of the scanner. An ultra-high resolution (UHR)-microPhantom was simulated with hot rods of different diameters $(0.35,0.40,0.45$, $0.50,0.60$ and $0.75 \mathrm{~mm}$ ), arranged into 6 segments within an acrylic cylinder [21]. For every segment, the spacing between adjacent rods was selected as twice the rod diameter as illustrated in Fig. 3a. The rod length of the phantom was selected as $2 \mathrm{~mm}$, smaller than the axial length of the scanner. We selected this value because our studies focused in the determination of the spatial resolution across the central transaxial plane. A shorter rod length allowed collection of sufficient number of events (to improve statistics) for a reasonable CPU time. The activity concentration was uniform with a total phantom activity of $8 \mathrm{MBq}$. For reference, one of the $0.75 \mathrm{~mm}$ diameter hot rods contained an activity of $400 \mathrm{kBq}$. Approximately 9 million coincidence events formed the raw sinogram prior to normalization. After FBP reconstruction, count profiles were extracted.

\section{Gap-filling algorithm}

Due to the dual-ended readout scheme of our scanner, each detector is separated at its entrance face by a $\sim 5 \mathrm{~mm}$ gap from its neighbors, resulting in a loss of a significant fraction of the sinogram data for a static-mode scanner. To illustrate the extent and shape of the gaps, Fig. 3b shows the normalized sinogram of the UHR microPhantom containing a $\beta^{+}\left[{ }^{18} \mathrm{~F}\right]$ source assuming a static scanner and a DOI resolution of $2 \mathrm{~mm}$, and Fig. $3 c$ displays the gapmask where the sinogram must be estimated (white regions). It can be observed that the gaps form a periodic pattern that varies along the radial and angular direction. In this particular case, the missing data in Fig. $3 b$ represent $\sim 46 \%$ of the region of the sinogram occupied by the object.

Different gap-filling methods have been proposed in the literature that either are applied in the sinogram space, such as bilinear interpolation, or that use the frequency domain properties of the sinogram data. Bilinear/bicubic interpolation methods work relatively well if the gaps are small or if their pattern does not vary along the angular direction [22,23]. However, when the gaps are large, model-based algorithms which apply data consistency conditions by using filters in the Fourier space $[24,25]$ or data adaptive filters in the discrete cosine transform (DCT) domain $[26,27]$ have better performance.

Given the large extent and the diamond-like pattern of the gaps produced by the static-mode small-animal PET scanner, we applied a gap-filling procedure based on the use of the DCT proposed by Tuna et al. [26,27]. The DCT is a real, invertible, linear transformation of a function expressed as a sum of cosines with different frequencies and amplitudes. The rationale behind this method relies on the fact that the frequency components of the object, and those related to the gap-pattern, are separated in the frequency domain. The DCT space has the advantage of providing high-energy compaction for highly correlated data (which means that the transformed object can be easily analyzed using a few lowfrequency components), as well as gap DCT coefficients that are associated with high-frequency components.

The gap-filling method requires the design of a filter in the DCT domain with binary pixel elements. This filter is used iteratively in the DCT space to estimate the data lying inside the gaps and to progressively update the sinogram in the region of the gaps. The whole procedure is schematically shown in Fig. 4, where $(s, \phi)$ represent radial distance and azimuthal angle, $p(s, \phi)$ is the sinogram of a $2 \mathrm{D}$ object $\mathrm{f}(x, y)$ and $P\left(\omega_{s}, \omega_{\phi}\right)$ is the two-dimensional DCT of $p(s, \phi)$ in the $\left(\omega_{s}, \omega_{\phi}\right)$ domain.

The DCT gap-filling method requires two important elements:

1. A filter in the DCT space designed using the sinogram of the object without gaps in order to isolate its coefficients from those that describe the gaps. In our case, for a given source distribution, the shape of the DCT filter was determined by the 2D-DCT sinogram of the source with a rotating-mode scanner. The shape of this filter is inspired in the original work of Karp et al. [24] who proposed a constrained Fourier space method for compensation of missing data using a bow-tie filter.

2. A gap-mask which defines the spatial distribution of the gaps in the sinogram space. In this case, a set of masks was obtained (one per DOI resolution) by segmenting the corresponding 


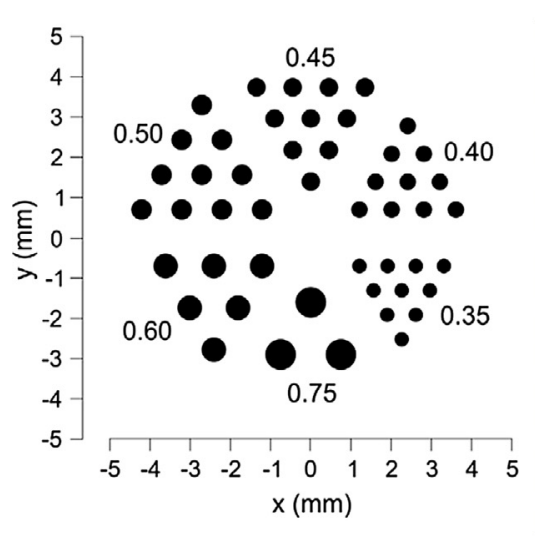

a)

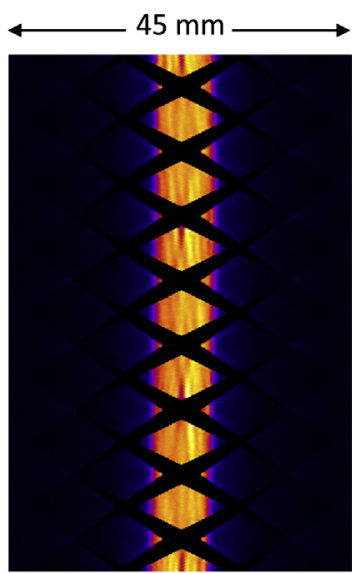

b)

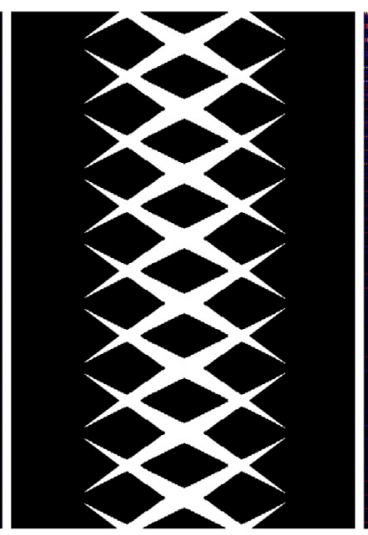

c)

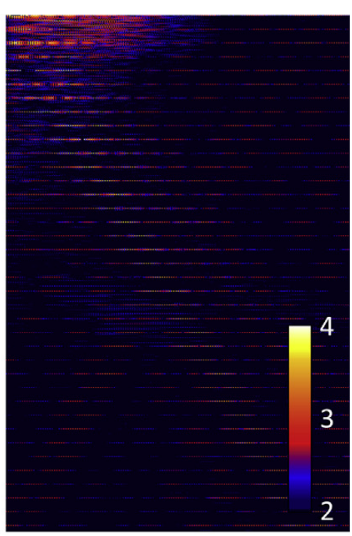

d)

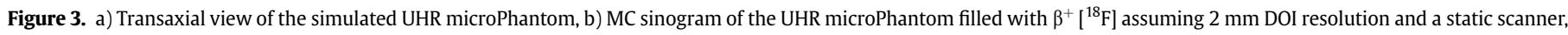
c) Gap-mask obtained by segmenting the normalization sinogram from the static-mode scanner, d) 2D-discrete cosine transform (DCT) of (b) in log10 scale.

simulated normalization sinograms of the static-mode scanner. Fig. $3 c$ shows the gap-mask for $2 \mathrm{~mm}$ FWHM ${ }_{\text {DOI }}$.

The gap-filling algorithm was terminated empirically after 10 iterations since no significant improvement in the sinograms and the reconstructed images was observed after that.

\section{Results}

\section{Reconstructed resolution}

The $\beta^{+}\left[{ }^{18} \mathrm{~F}\right]$ point source sinograms of the rotating small-animal scanner at 2, 6 and $12 \mathrm{~mm}$ off-center assuming $2 \mathrm{~mm}$ DOI resolution are shown in Fig. 5, together with their 2D-DCT coefficients and their DCT filters in the DCT domain with unity values within the white areas and zero otherwise. The design of the DCT filters for the point sources required a careful examination of their 2D-DCT coefficients; the filters showed simple-quadrilateral shapes with four vertices: $(0,0),\left(0, \omega_{\phi 1}\right),\left(\omega_{s}, 0\right)$ and $\left(\omega_{s}, \omega_{\phi 2}\right)$, the main difference between the filters was determined by their $\omega_{\phi 2}$ value. It was observed that the larger the radial distance of the point source, the larger the value of $\omega_{\phi 2}$. A plot of $\omega_{\phi 2}$ as a function of radial distance of the point sources indicates a linear relationship (not shown). This dependence can be used as guidance to estimate the filter shape for any object whose radial distance extension is known.

The sinograms of the static scanner are also displayed in Fig. 5; the large fraction of data lost due to the presence of gaps is evident. The application of the gap-filling method after 10 iterations

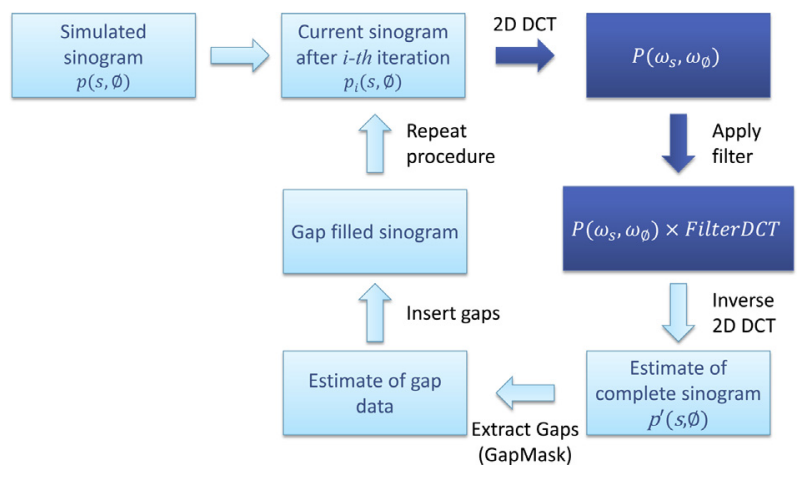

Figure 4. Flow diagram of the iterative DCT gap-filling algorithm. allowed the recovery of the missing data, with better performance for point sources at radial distances $\leq 6 \mathrm{~mm}$.

Figure 6 shows the reconstructed images of the $\beta^{+}\left[{ }^{18} \mathrm{~F}\right]$ point sources and count profiles (radial and tangential) across their maximum intensity for all the simulated positions for the static, static with gaps-filled and rotating scanners. The rotating scanner produces practically streak-free symmetric images, with a slight elongation of the point source as the radial distance increases, as expected. Conversely, the static scanner produces noticeable streak artifacts, with distorted images. The streak artifacts are reduced after the application of the DCT gap-filling method, improving image quality with better symmetry, showing a close resemblance to those from the rotating scanner.

The tangential and radial resolutions for the scanning modes are plotted in Fig. 7. When the DCT gap filling method is applied to the static-mode data, the trend of the radial spatial resolution is closer to the rotating scanner data, showing overall good agreement within their statistical uncertainties. There is a tendency of the static gaps-filled results to show a degradation of the tangential spatial resolution for all radial distances. Conversely, the radial spatial resolution reverses this tendency as the radial offset of the source increases, probably due to the inability of the DCT filter to completely fill the gaps at large offset distances which causes artifacts that can distort the FWHM estimates. Also of note is that the radial resolution for the static-mode data is apparently superior at large radial offsets. However, this is an effect caused by the major streaking artifacts which can artificially improve the spatial resolution along certain directions.

While the tangential spatial resolution has a weak dependence with source position and DOI resolution, the radial spatial resolution shows a moderate dependence with these variables for the values assumed in this work. The FWHM radial resolution of the static gaps-filled scanner ranged from $0.66 \mathrm{~mm}$ at the center of the FOV to $0.83 \mathrm{~mm}$ at a radial offset of $14 \mathrm{~mm}$ for $2 \mathrm{~mm}$ DOI resolution; this range extended from 0.66 to 0.99 for the same radial offsets when using a $3 \mathrm{~mm}$ DOI resolution.

In order to examine the effect of positron range and photon noncolinearity in the performance of the small-animal PET scanner, Table 1 summarizes the tangential and radial spatial resolution results for all source locations, DOI resolutions, source types and scanning modes. As expected, the spatial resolution estimated with the anti-parallel $511 \mathrm{keV}$ gammas is always better than the one predicted with $\left[{ }^{18} \mathrm{~F}\right]$ positrons. The data indicate that the resolution degrades by an average of about $170 \mu \mathrm{m}$ when considering all radial 

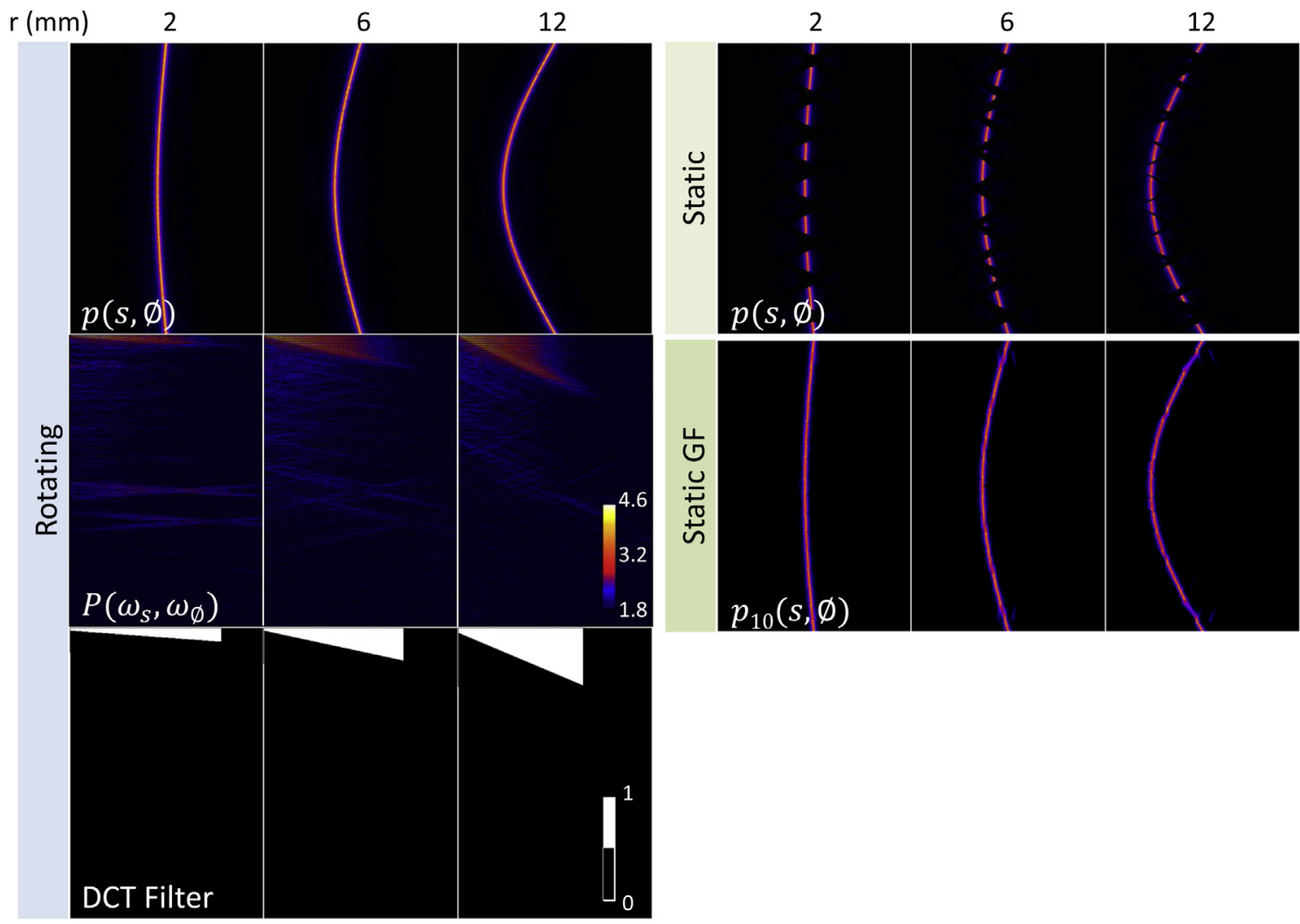

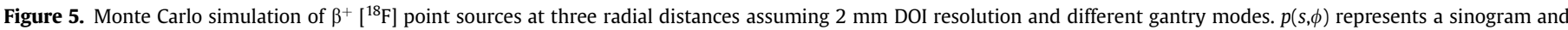
$p_{10}(s, \phi)$ is the gaps-filled (GF) sinogram after 10 iterations the 2D-DCT coefficients, $P\left(\omega_{s}, \omega_{\phi}\right)$, are shown in log10 scale.

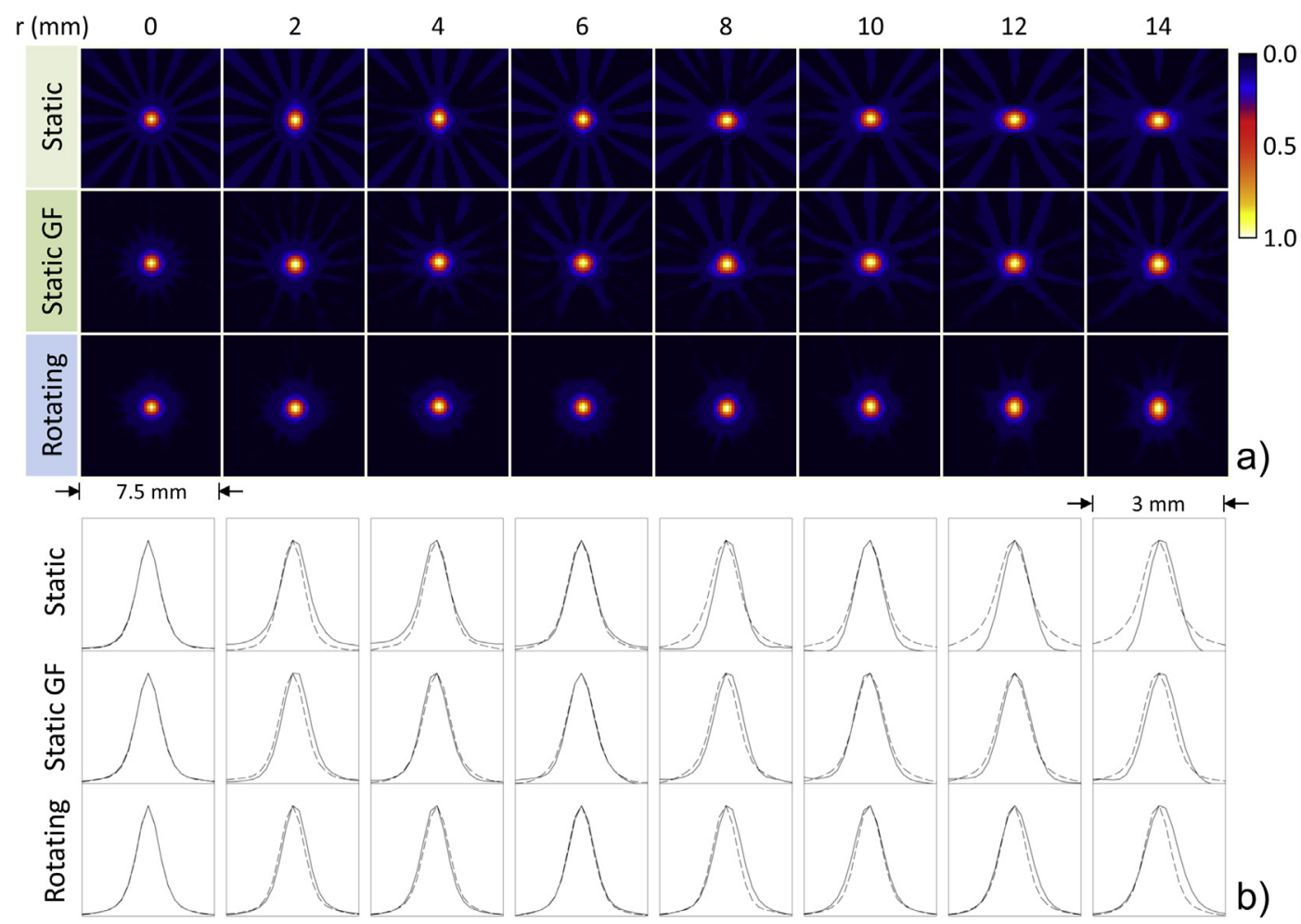

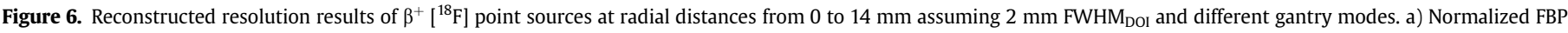
images. b) Radial (solid) and tangential (dashed) profiles at the maximum intensities. Note that the distance scales in a) and b) are different. 


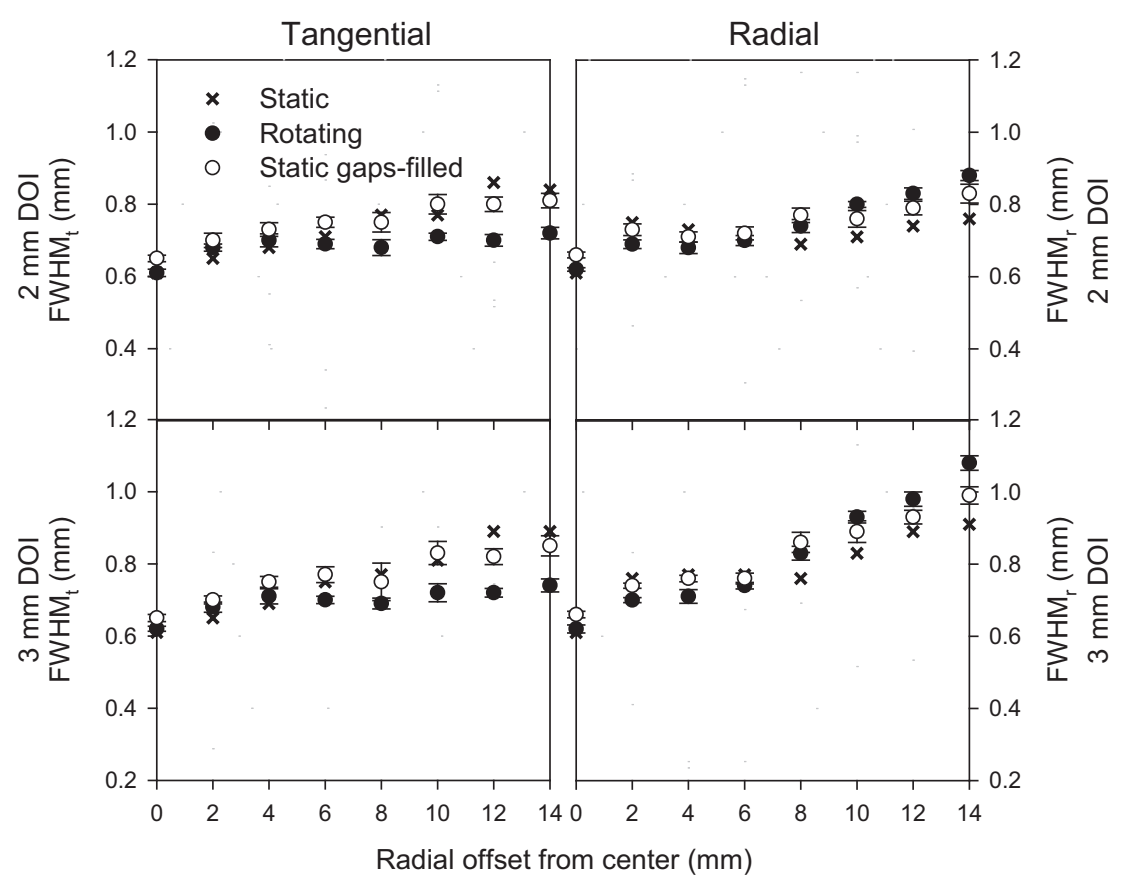

Figure 7. Tangential $\left(\mathrm{FWHM}_{\mathrm{t}}\right)$ and radial $\left(\mathrm{FWHM}_{\mathrm{r}}\right)$ spatial resolutions for $\beta^{+}\left[{ }^{18} \mathrm{~F}\right]$ point sources as a function of radial distance, assuming 2 and $3 \mathrm{~mm}$ DOI resolutions.

offsets. Assuming resolution components can be added in quadrature, this suggests that the combined contribution due to positron range $\left({ }^{18} \mathrm{~F}\right)$ and non-colinearity for this scanner design is about 420-480 $\mu \mathrm{m}$. For example, Table 1 shows that for $2 \mathrm{~mm}$ DOI resolution the average radial resolution for two anti-parallel $511 \mathrm{keV}$ photons $\left(\mathrm{FWHM}_{\mathrm{radial}}^{\gamma-\gamma} \mathrm{s}\right)$ is $0.60 \mathrm{~mm}$, while the average radial resolution for $\left[{ }^{18} \mathrm{~F}\right]$ positrons $\left(\mathrm{FWHM}_{\text {radial }}^{\beta^{+}}\right)$is $0.75 \mathrm{~mm}$. If the resolution components can be added in quadrature then:

$\mathrm{FWHM}_{\text {radial }}^{\beta^{+}}=\sqrt{\left(\mathrm{FWHM}_{\text {radial }}^{\gamma-\gamma}\right)^{2}+\left(\mathrm{FWHM}_{\text {radial }}^{\mathrm{R}+\mathrm{NC}}\right)^{2}}$

where $\mathrm{FWHM}_{\text {radial }}^{\mathrm{R}+\mathrm{NC}}$ is the contribution due to positron range and non-colinearity. This is estimated to be:
$\mathrm{FWHM}_{\text {radial }}^{\mathrm{R}+\mathrm{NC}}=\sqrt{(0.75)^{2}-(0.60)^{2}}=0.450 \mathrm{~mm}$

\section{Image resolution phantom}

Figure 8 displays the sinogram of the UHR microPhantom containing a $\beta^{+}\left[{ }^{18} \mathrm{~F}\right]$ source assuming a rotating scanner (a), the sinogram's 2D-DCT (b) and the DCT filter with unity values within the white area and zero otherwise (c). This filter was estimated on the basis of the filters designed for the point sources, in particular the one at $4 \mathrm{~mm}$ radial distance that corresponds to the radial extent of this phantom; it can be observed that its shape is in accordance with the distribution of the DCT coefficients shown in

Table 1

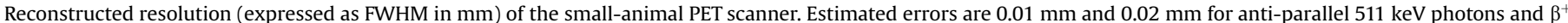
$\left[{ }^{18} \mathrm{~F}\right]$ sources, respectively.

\begin{tabular}{|c|c|c|c|c|c|c|c|c|}
\hline \multirow[t]{3}{*}{$\mathrm{r}(\mathrm{mm})$} & \multicolumn{4}{|c|}{ Static scanner gaps-filled } & \multicolumn{4}{|c|}{ Rotating scanner } \\
\hline & \multicolumn{2}{|c|}{$2 \mathrm{~mm}$ DOI } & \multicolumn{2}{|c|}{$3 \mathrm{~mm}$ DOI } & \multicolumn{2}{|c|}{$2 \mathrm{~mm}$ DOI } & \multicolumn{2}{|c|}{$3 \mathrm{~mm}$ DOI } \\
\hline & Radial & Tangential & Radial & Tangential & Radial & Tangential & Radial & Tangential \\
\hline \multicolumn{9}{|c|}{ Two anti-parallel $511 \mathrm{keV}$ photons } \\
\hline 0 & 0.50 & 0.50 & 0.48 & 0.48 & 0.48 & 0.48 & 0.48 & 0.48 \\
\hline 2 & 0.58 & 0.53 & 0.55 & 0.52 & 0.53 & 0.51 & 0.55 & 0.52 \\
\hline 4 & 0.58 & 0.56 & 0.56 & 0.55 & 0.54 & 0.53 & 0.57 & 0.54 \\
\hline 6 & 0.56 & 0.58 & 0.58 & 0.54 & 0.53 & 0.53 & 0.58 & 0.54 \\
\hline 8 & 0.61 & 0.58 & 0.67 & 0.52 & 0.60 & 0.52 & 0.67 & 0.52 \\
\hline 10 & 0.62 & 0.64 & 0.74 & 0.58 & 0.65 & 0.54 & 0.77 & 0.54 \\
\hline 12 & 0.65 & 0.64 & 0.77 & 0.63 & 0.67 & 0.55 & 0.83 & 0.56 \\
\hline 14 & 0.68 & 0.67 & 0.88 & 0.62 & 0.73 & 0.56 & 0.93 & 0.56 \\
\hline $\begin{array}{l}\text { Average } \\
\beta^{+}\left[{ }^{18} \mathrm{~F}\right]\end{array}$ & 0.60 & 0.59 & 0.65 & 0.56 & 0.59 & 0.53 & 0.67 & 0.53 \\
\hline 0 & 0.66 & 0.65 & 0.66 & 0.65 & 0.62 & 0.61 & 0.62 & 0.62 \\
\hline 2 & 0.73 & 0.70 & 0.74 & 0.70 & 0.69 & 0.68 & 0.70 & 0.68 \\
\hline 4 & 0.71 & 0.73 & 0.76 & 0.75 & 0.68 & 0.70 & 0.71 & 0.71 \\
\hline 6 & 0.72 & 0.75 & 0.76 & 0.77 & 0.70 & 0.69 & 0.74 & 0.70 \\
\hline 8 & 0.77 & 0.75 & 0.86 & 0.75 & 0.74 & 0.68 & 0.83 & 0.69 \\
\hline 10 & 0.76 & 0.80 & 0.89 & 0.83 & 0.80 & 0.71 & 0.93 & 0.72 \\
\hline 12 & 0.79 & 0.80 & 0.93 & 0.82 & 0.83 & 0.70 & 0.98 & 0.72 \\
\hline 14 & 0.83 & 0.81 & 0.99 & 0.85 & 0.88 & 0.72 & 1.08 & 0.74 \\
\hline Average & 0.75 & 0.75 & 0.82 & 0.77 & 0.74 & 0.69 & 0.82 & 0.70 \\
\hline
\end{tabular}




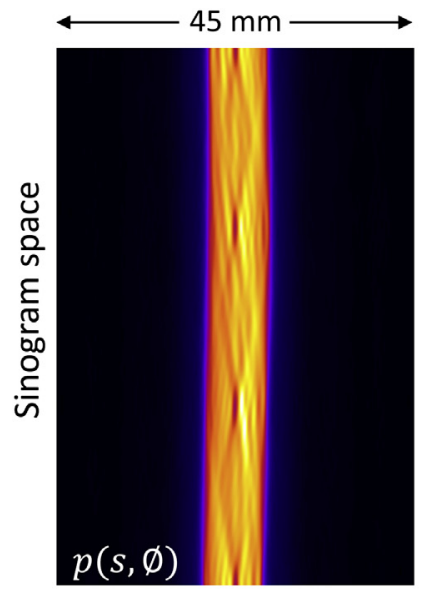

a)

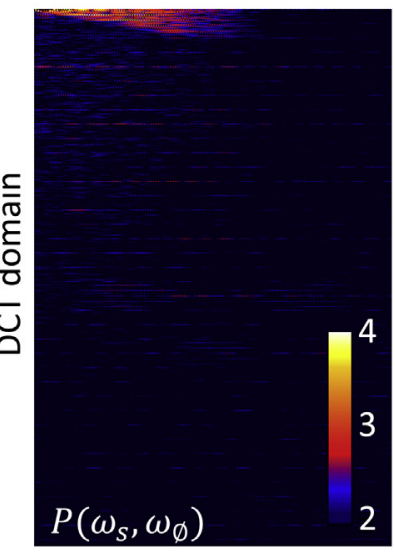

b)

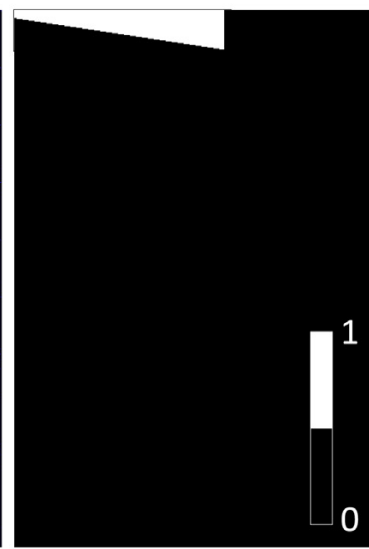

c)

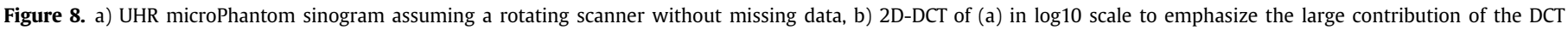
coefficients at low frequencies, c) DCT filter.

Fig. 8b. For comparison purposes, the 2D-DCT sinogram of the UHR microPhantom with a static scanner is displayed in Fig. 3d, clearly showing the difficulty of isolating the coefficients associated with the object from those that describe the gaps.

Figure 9 shows the results of the UHR microPhantom assuming $2 \mathrm{~mm}$ DOI resolution and different combinations of scanner-modes and source types. In all cases, the rotating scanner produced the best artifact-free image quality in terms of spatial resolution and contrast. The results from a static scanner show the missing data in the sinograms which in turn produce reconstructed images with severe streak and ring artifacts and high levels of noise, as shown from the extracted count profiles across the $0.75,0.5$ and $0.45 \mathrm{~mm}$ rods. However, when the DCT gap-filling algorithm is applied to this set of data, the gaps-filled sinograms, the reconstructed images and the corresponding count profiles are very similar to those produced with data from a rotating scanner. In both cases, static gaps-filled and rotating scanners, the quality of the reconstructed images is excellent. a)
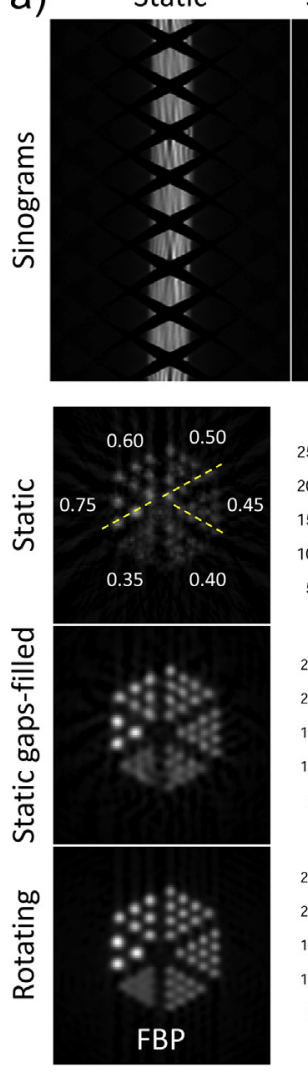

Static gaps-filled
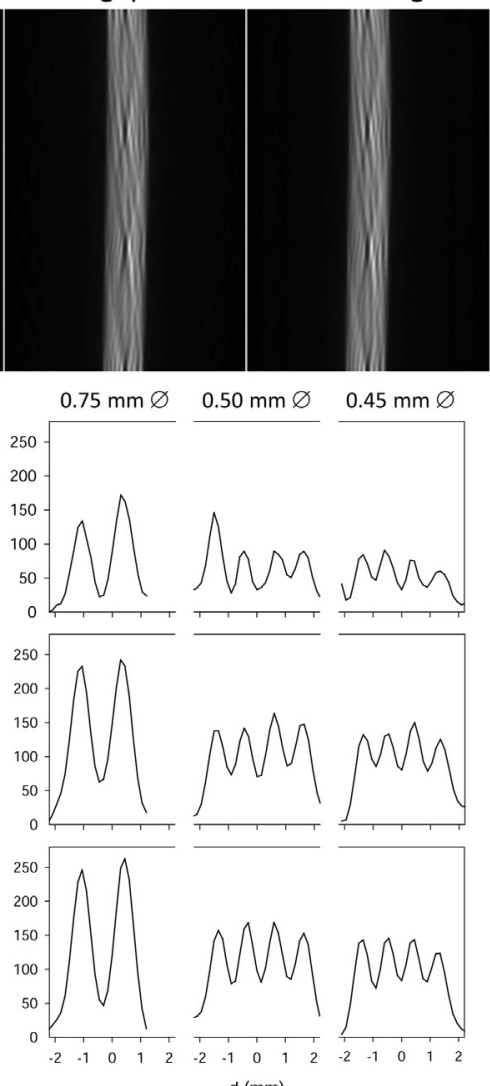

b)

Static
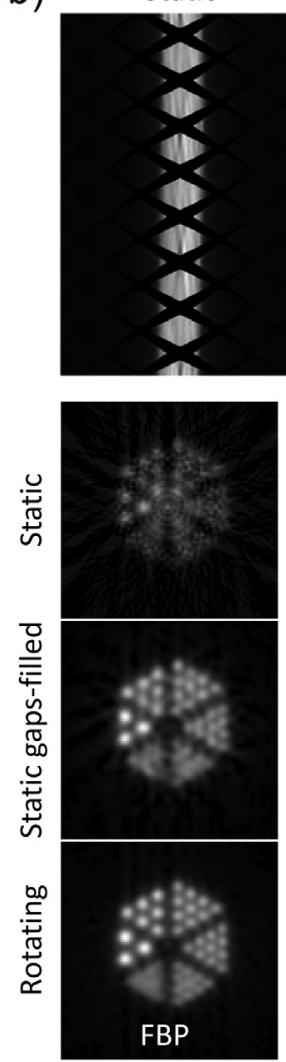

$\mathrm{d}(\mathrm{mm})$
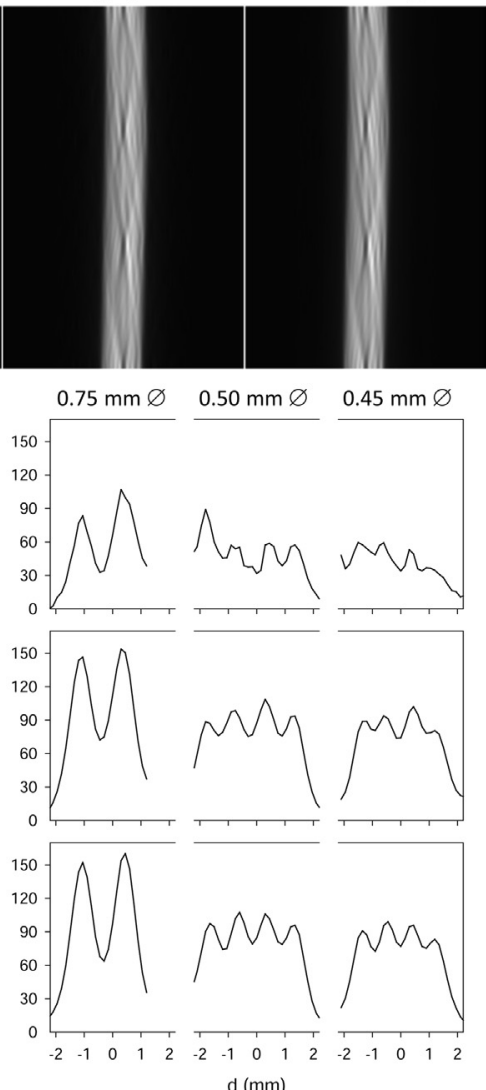

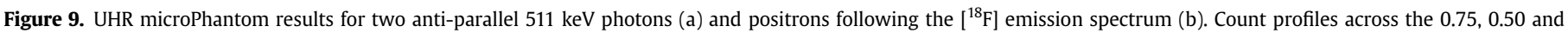
$0.45 \mathrm{~mm}$ rods are shown in all cases. 
The effect of the positron range can be observed in Fig. 9a and b that show the simulations for anti-parallel $511 \mathrm{keV}$ photons and positrons, respectively. The comparison between the count profiles shown in the same figure clearly displays the image blurring produced by the use of a $\beta^{+}\left[{ }^{18} \mathrm{~F}\right]$ source which also reduces contrast in the count profiles. To illustrate the contrast reduction due to positron range, peak-to-valley ratios were calculated from the count profiles of the rotating scanner. The simulation of anti-parallel $511 \mathrm{keV}$ photons produced average peak-to-valley ratios of 5.47, 1.95 and 1.73 for the $0.75,0.50$ and $0.45 \mathrm{~mm}$ rods, respectively, while the corresponding values for positrons were $2.45,1.30$ and 1.18 . These figures indicate a stronger effect for the larger diameter rods.

For anti-parallel $511 \mathrm{keV}$ photons, using either static gaps-filled or rotating gantry modes, hot rods with a diameter of $0.4 \mathrm{~mm}$ were resolved. For $\beta^{+}\left[{ }^{18} \mathrm{~F}\right]$ and rotational gantry motion, rods with a diameter of 0.45 were resolved, whilst with a static gaps-filled gantry the smallest resolvable rods had $0.5 \mathrm{~mm}$ diameter.

\section{Discussion and conclusions}

The Monte Carlo simulation of a prototype small-animal PET scanner designed for mouse brain imaging studies provided valuable information regarding the transaxial spatial resolution that can be expected from the system. The simulation of the rotating scanner served two aims. First, it allowed the generation of gap-free sinograms that were used to predict the best spatial resolution that the scanner could achieve when using the FBP method prescribed in the NEMA standard, without the inclusion of possible effects due to data manipulation to fill the gaps. Both, the tangential and radial spatial resolution for $\beta^{+}\left[{ }^{18} \mathrm{~F}\right]$ point sources ranged from $0.61 \mathrm{~mm}$ to $0.74 \mathrm{~mm}$ for source positions $\leq 6 \mathrm{~mm}$ from the center and either 2 or $3 \mathrm{~mm}$ $\mathrm{FWHM}_{\mathrm{DOI}}$. The image quality of the UHR microPhantom was excellent, without streak or ring artifacts. In this case, when using a positron $\left[{ }^{18} \mathrm{~F}\right]$ source, hot rods as small as $0.5 \mathrm{~mm}$ were clearly distinguishable, as shown by the extracted count profiles. Second, the gap-free data were used to design the DCT filter required by the gapfilling algorithm implemented in the static mode case. The DCT filters had simple-quadrilateral shapes: the determination of these filters for the point sources as a function of radial distance was extremely useful and served as a basis to estimate the DCT filter for the UHR microPhantom, only by knowing its radial extent. This means that once the DCT filters for point sources are obtained using gap-free sinograms, they can be used to design the filters for extended objects.

The results from the static scanner showed the extent and the pattern produced by the gaps between the detectors in the sinograms. As expected, the reconstructed images were distorted, with significant streak and ring artifacts. When the gaps were filled using the DCT algorithm, the artifacts were significantly reduced, substantially improving the quality of the reconstructions of point sources. The static gaps-filled sinograms of $\beta^{+}\left[{ }^{18} \mathrm{~F}\right]$ point sources reconstructed with FBP produced tangential and radial spatial resolutions ranging from $0.65 \mathrm{~mm}$ to $0.77 \mathrm{~mm}$ for source positions $\leq 6 \mathrm{~mm}$ from the center

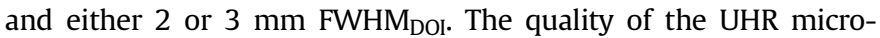
Phantom was also very good; hot rods of $0.5 \mathrm{~mm}$ were also resolved, although in this case the count profiles showed lower contrast than those from the rotating scanner. In all cases, a slight resolution loss was observed with respect to the results obtained with a rotating scanner, probably due to the elimination of the very high frequency components of the object during the gap-filling procedure. In spite of this, the DCT gap-filling algorithm had excellent performance.

The visual inspection of the sinograms and the reconstructed images, together with the quantification of the radial and tangential spatial resolutions, indicate that the DCT gap-filling method is an acceptable approximation for sources at radial distances $\leq 6 \mathrm{~mm}$, establishing a limit on the FOV of $12 \mathrm{~mm}$ that can be covered when using a static scanner together with the DCT gap-filling method. Since the width of the brain of an eight-week-old male 129S1/SvImJ mice is $\sim 8 \mathrm{~mm}$ [28], the restricted FOV does not represent a drawback in the application that we intend for our scanner. Additionally, for this $12 \mathrm{~mm}$ FOV, the predicted spatial resolutions for the static gaps-filled and rotating scanners are, within the uncertainty of the simulations and methods, the same. This indicates that it is not necessary to design and engineer a rotating gantry for the scanner currently under construction and that it should be feasible to produce quantitative data with a static scanner and an analytic FBP reconstruction as long as compensation of missing data is applied using the DCT algorithm. This is important as it means that standard NEMA resolution protocols can be used with the scanner without rotation once it is constructed.

Our calculations indicate that the combined effect of the positron range of $\left[{ }^{18} \mathrm{~F}\right]$ and photon non-colinearity is a blurring of $\sim 450 \mu \mathrm{m}$ assuming that the combination of these factors can be approximated as a Gaussian and computed by subtracting the results for the ideal $511 \mathrm{keV}$ photons from the ${ }^{18} \mathrm{~F}$ positron source in quadrature. Levin and Hoffman (1999) reported $\beta^{+}\left[{ }^{18} \mathrm{~F}\right]$ positron annihilation point distributions in water with a cusp-like shape and FWHM and FWTM of $0.102 \mathrm{~mm}$ and $1.03 \mathrm{~mm}$, respectively. These authors also predicted that for ${ }^{18} \mathrm{~F}$, an infinitely thin detector and a $20 \mathrm{~cm}$ diameter scanner, the system spatial resolution would reach a limit of $0.6 \mathrm{~mm}$ FWHM due to positron range, photon non-colinearity and detector element size effects. Stickel and Cherry [2] predicted that for ${ }^{18} \mathrm{~F}$ in a scanner with $0.45 \mathrm{~mm}$ detector elements, and a ring diameter of $8 \mathrm{~cm}$, a resolution as high as $0.55 \mathrm{~mm}$ FWHM could be obtained. The results of the present study, which unlike prior studies incorporated DOI effects, indicate that the prototype static DCT gaps-filled scanner with $0.45 \mathrm{~mm}$ crystal size at the front, $61 \mathrm{~mm}$ bore, $2-3 \mathrm{~mm}$ DOI encoding accuracy and radial source positions $\leq 6 \mathrm{~mm}$ leads to a system spatial resolution of $0.70 \pm 0.05 \mathrm{~mm}$ FWHM; this value includes positron range and photon non-colinearity effects, and represents the average of $2 \mathrm{~mm}$ and $3 \mathrm{~mm} \mathrm{DOI}$, and radial and tangential resolutions. Our results are consistent with these earlier studies of somewhat different geometries that also used different MC codes.

The results obtained in this work show the importance of applying an appropriate gap-filling method to evaluate the reconstructed spatial resolution attained by a scanner that generates sinograms with significant gaps. A similar situation applies for many scanners proposed or developed by different research groups working worldwide. As stated by the current NEMA protocol for small-animal PET, the evaluation of the reconstructed resolution of point sources must be done using FBP, therefore it is important to have robust FBP-based methods available for the spatial resolution evaluation of this and other scanners. It was found that the determination of the DCT filters for point sources at different radial distances can serve as guideline to estimate the DCT filters for extended sources. This can be of particular importance not only in MC simulation studies, but also when the gap-filling procedure is applied to practical real experiments.

\section{Acknowledgments}

This work was funded by NIH grant R01 EB006109. M. Rodríguez acknowledges DGAPA-UNAM for financial support during her sabbatical leave.

\section{References}

[1] Larobina $M$, Brunetti A, Salvatore $M$. Small animal PET: a review of commercially available imaging systems. Curr Med Imag Rev 2006;2:187-92.

[2] Stickel J, Cherry SR. High-resolution PET detector design: modelling components of intrinsic spatial resolution. Phys Med Biol 2005;50:179-95. 
[3] Moehrs S, Del Guerra A, Herbert DJ, Mandelkern MA. A detector head design for small-animal PET with silicon photomultipliers (SiPM). Phys Med Biol 2006;51:1113-27.

[4] Spanoudaki V, Torres-Espallardo I, Rafecas M, Ziegler S. Performance evaluation of MADPET-II, a small animal dual layer LSO-APD PET scanner with individual detector read out and depth of interaction information. J Nucl Med 2007;48(Suppl. 2):131.

[5] Tétrault MA, Viscogliosi N, Riendeau J, Bélanger F, Michaud JB, Semmaoui H, et al. System architecture of the LabPET small animal PET scanner. IEEE Trans Nucl Sci 2008;55:2546-50.

[6] Li X, Hunter WCJ, Lewellen TK, Miyaoka RS. Design of a trapezoidal slat crystal (TSC) PET detector for small animal PET/MR imaging. IEEE Nucl Sci Symp Conf Rec 2010:2598-601.

[7] Gu Y, Matteson JL, Skelton RT, Deal AC, Stephan EA, Duttweiler F, et al. Study of a high-resolution, 3D positioning cadmium zinc telluride detector for PET. Phys Med Biol 2011;56:1563-84.

[8] Llosá G, Barrio J, Cabello J, Lacasta C, Oliver JF, Rafecas M, et al. Development of a PET prototype with continuous LYSO crystals and monolithic SiPM matrices. IEEE Nucl Sci Symp Conf Rec 2011:3631-4.

[9] Yamamoto S, Watabe H, Hatazawa J. Performance comparison of Si-PM-based block detectors with different pixel sizes for an ultrahigh-resolution small animal PET system. Phys Med Biol 2011;56:N227-36.

[10] Yang Y, Wu YB, Qi J, St James S, Du HN, Dokhale PA, et al. A prototype PET scanner with DOI-encoding detectors. J Nucl Med 2008;49:1132-40.

[11] Yang Y, St James S, Wu Y, Du HN, Qi J, Farrell R, et al. Tapered LSO arrays for small animal PET. Phys Med Biol 2011;56:139-53.

[12] Stickel JR, Qi J, Cherry SR. Fabrication and characterization of a $0.5-\mathrm{mm}$ lutetium oxyorthosilicate detector array for high-resolution PET applications. J Nucl Med 2007;48:115-21.

[13] St James S, Yang YF, Wu YB, Farrell R, Dokhale P, Shah KS, et al. Experimenta characterization and system simulations of depth of interaction PET detectors using $0.5 \mathrm{~mm}$ and $0.7 \mathrm{~mm}$ LSO arrays. Phys Med Biol 2009;54:4605-19.

[14] St James S, Yang Y, Bowen SL, Qi J, Cherry SR. Simulation study of spatia resolution and sensitivity for the tapered depth of interaction PET detectors for small animal imaging. Phys Med Biol 2010;55:N63-74.
[15] Defrise M, Kinahan PE, Michel CJ. Image reconstruction algorithms in PET. In: Bailey DL, Townsend DW, Valk PE, Maisey MN, editors. In positron emission tomography. London: Basic Sciences Springer; 2005.

[16] Performance measurements of small animal positron emission tomographs. Rosslyn, VA: National Electrical Manufacturers Association; 2008. NEMA Standards Publication NU-4-2008.

[17] Levin CS, Hoffman EJ. Calculation of positron range and its effect on the fundamental limit of positron emission tomography system spatial resolution. Phys Med Biol 1999;44:781-99.

[18] Cherry SR, Sorenson J, Phelps M. Physics in nuclear medicine. Philadelphia: Saunders; 2003.

[19] Jan S, Santin G, Strul D, Strul D, Staelens S, Assié K, Autret D, et al. GATE: a simulation toolkit for PET and SPECT. Phys Med Biol 2004;49:4543-61.

[20] Yang Y, Tai YC, Siegel S, Newport DF, Bai B, Li Q et al. Optimization and performance evaluation of the microPET II scanner for in vivo small-animal imaging. Phys Med Biol 2004;49:2527-45.

[21] Beekman F, Van der Have F. The pinhole: gateway to ultra-high-resolution three-dimensional radionuclide imaging. Eur J Nucl Med Mol 2007;34:151-61.

[22] De Jong HWAM, Boellaard R, Knoess C, Lenox M, Michel C, Casey C, et al. Correction methods for missing data in sinograms of the HRRT PET scanner. IEEE Trans Nucl Sci 2003;50:1452-6.

[23] Loukiala A, Tuna U, Beer S, Jahnke S, Ruotsalainen U. Gap-filling methods for 3D PlanTIS data. Phys Med Biol 2010;55:6125-39.

[24] Karp JS, Muehllener G, Lewitt RM. Constrained Fourier space method for compensation of missing data in emission computed tomography. IEEE Trans Med Imag 1988;7:21-5.

[25] Ren R, Li Q Ahn S, Cho S, Leahy RM. Estimation of gap data using bow-tie filters for 3D time-of-flight PET. IEEE Nucl Sci Symp Conf Rec 2010:2691-4.

[26] Tuna U, Peltonen S, Ruotsalainen U. Data estimation for the ECAT HRRT sinograms by utilizing the DCT domain. IEEE Nucl Sci Symp Conf Rec 2008:5076-80.

[27] Tuna U, Peltonen S, Ruotsalainen U. Gap-filling for the high-resolution PET sinograms with a dedicated DCT-domain filter. IEEE Trans Med Imag 2010;29:830-9.

[28] Kovacevic N, Henderson JT, Chan E, Lifshitz N, Bishop J, Evans AC, et al. A Three-dimensional MRI atlas of the mouse brain with estimates of the average and variability. Cereb Cortex 2005;15:639-45. 